disease, which has been sufficiently dwelt upon before, I may remark that the facts here stated establish strong presumption that, as bleeding was less frequently practised, the character of diseases had undergone material difference.

Much more might be said on the manner in which bleeding acts in such cases; and I cannot think that it is satisfactorily accounted for simply by the removal of a portion of the blood, as seems to be the prevailing opinion at present. I will now, however, simply say,

$$
\text { "Mrulta renascentur quæ jam cecidere." }
$$

\section{ON THE EMPLOYMENT OF LARGE DOSES OF IODIDE OF POTASSIUM IN SEVERE} FORMS OF TERTIARY SYPHILIS.

By Henry Smrth, F.R.C.S., Assistant-Surgeon to King's College Hospital.

THE subjoined case was in the hospital under my care last summer. It attracted considerable attention at the time, partly from the extraordinary severity of the symptoms, but mainly in consequence of the rapidity of the cure under the influence of very large doses of iodide of potassium, when smaller doses of the same medicine had repeatedly failed to be of any service. Since the patient's dismissal, he has every now and then shewn himself at the hospital, and he continues quite well.

A tall well-made labouring man, aged 44, was admitted on July 24th, 1865 . He was so terribly disfigured, that none of his features were discernible, the whole of his face being covered with a mask made of several pieces of linen. When these had been removed, the entire of the face with the exception of a part in front of the left ear, was one mass of deep unhealthy ulceration, presenting the well marked character of tertiary syphilitic ulceration of the skin. The poor man was a most hideous object, and was in a very weak state of health. There was, in addition, a large unhealthy ulcer over the right external malleolus leading down to bare bone.

It appears that he had the primary ulcer twenty years previously; this had been succeeded from time to time by various secondary and tertiary manifestations, which were remedied by appropriate treatment; but during the last three years this ulceration in the face had been present, and had defied all treatment. He had been under treatment at the hands of several hospital surgeons, and just previous to his coming to me had been an inmate for four months at one of the hospitals. He had taken a large quantity amongst other things of iodide of potassium; but he informed me that he had never used it in larger doses than five grains.

I had him confined to bed, ordered him generous nourishment, and prescribed twenty grains of iodide of potassium to be taken thrice daily. I must confess I had little hope of doing better than others with this poor man; but he had not taken the remedy more than a week, when manifest signs of improvement presented themselves. Some of the ulcerations began to diminish in size, his appetite improved, and no bad effects of so large a dose were observable. I then ordered the dose to be increased to thirty grains; and in two weeks the ulceration had half healed; he continued with the same, and at the termination of six weeks the whole of the ulcers had firmly and thoroughly healed, the man's appetite and strength had vastly improved, and the ulcer on the leg had much contracted. I dismissed him with injunctions to continue the medicine, which he did for some weeks longer, when he presented himself per- fectly well in every respect. I saw this man last week, and he remains quite well.

This man was exhibited at the meeting of the Medical Society of London, when a large number of surgeons were present to hear the views of Dr. Boeck on syphilisation. I was glad to hear one or two of the speakers confirm my views as to the value of large doses of iodide of potassium, when smaller doses had failed. The pupils at King's College Hospital have, for some years past, been made familiar with the excellent results of the use of iodide of potassium in scruple-doses for severe tertiary ulcerations of the skin and affections of the bone. It appears to me, after a very careful investigation and much practical experience, that iodide of potassium is not very useful in curing the ordinary secondary forms of syphilis; but that when the disorder has shown itself in the more severe tertiary forms, as in ulcerations of the skin, large and painful nodes, affections of the hard palate and nose, the remedy in question is of the utmost value, when freely employed; but I think the case detailed especially shows the importance of giving very large doses in instances where the ordinary doses have completely failed to give any relief whatever. We must, of course, look out for the possibility and probability of iodism; should this occur, we need not give up the remedy altogether, but use it in smaller doses, and gradually increase it, until we have obtained a decided effect upon the symptoms. As an illustration of this, and as an example of the great value of iodide of potassium in a very intractable form of primary venereal ulceration, I will refer to a case recently under my care at King's College Hospital. It was that of an elderly man, who presented himself with an extensive serpiginous ulceration of the penis, which had commenced on the glans six months previously, and partially healing there, had extended over the whole circumference of the organ, destroying the prepuce, part of the scrotum, and showing no symptoms of abating. A peculiar characteristic of this ulcer, was the severe pain experienced by the patient both night and day.

This form of ulceration is by no means common, and amongst the large number of venereal cases met with at our hospital, I see but a case now and then. Hitherto, remedies seem to have been useless in stopping this ulceration; rest, time, and good nourishment, appear to have been the only effectual means. Iron, even in these slow but destructive cases, is useless.

Having hitherto failed to give any relief in such cases, and recognising the similarity between this and some of the ordinary tertiary ulcerations, I determined to try iodide of potassium in large doses, and accordingly ordered the patient to take ten grains three times daily. The patient came in four days quite iodised, but although the appearance of this sore was no better, the pain was greatly relieved. I therefore reduced the iodide of potassium to five grains three times daily. Iodism was no longer produced. In the course of a few days the sore began to heal, the pain entirely left him, cicatrisation went on steadily and surely, and, at the termination of one month, the man presented himself to notice with this large sore, which had resisted all sorts of treatment for six months, firmly healed.

16, Caroline Street, Bedford Square.

VICE-VERSA. In England, where any one may sell drugs, there is a cry out against the liberty of sale, whereby through ignorance many persons are yearly poisoned. In France, where only pharmaciens are allowed to sell medicines, there is a loud cry raised against the restriction. 